\title{
Dimension Spreading for Coherent Opportunistic Communications
}

\author{
Jordi Borràs ${ }^{1}$, Josep Font-Segura ${ }^{2}$, Jaume Riba ${ }^{1}$, and Gregori Vázquez ${ }^{1}$ \\ ${ }^{1}$ Department of Signal Theory and Communications, Technical University of Catalonia (UPC) \\ D5-\{218A, 116, 204\}, Campus Nord UPC, Jordi Girona 1-3, 08034 Barcelona, Spain \\ ${ }^{2}$ Department of Information and Communication Technologies, Universitat Pompeu Fabra (UPF) \\ Edifici Tànger 55.204, Roc Boronat 138, 08018 Barcelona, Spain \\ Email: jordi.borras.pino@upc.edu, josep.font@ieee.org, \{jaume.riba, gregori.vazquez\}@upc.edu
}

\begin{abstract}
The waveform optimization problem for opportunistic communications is addressed, based on sensing the secondorder statistics of the existing transmissions. We propose a minimum-norm waveform optimization that exhibits robustness to the worst-case subspace mismatch, minimizes the spectral overlapping with the existing transmissions, is rotationally invariant, and has maximally white spectrum. The derived solution can be seen as a different kind of signal dimension-based spreading. In addition, the effects of the residual interference caused to the existing transmissions are studied. Numerical results are provided to assess the performance of the proposed solution in the frequency domain for the asymptotic case. The level of induced interference is compared to traditional null space techniques.

Index Terms-Opportunistic communication, waveform optimization, dimension spreading, distributed networks.
\end{abstract}

\section{INTRODUCTION}

The increasing demand for high data-rate services intensifies the interest of spectral efficiency. Since the spectrum is a limited resource, techniques such as cognitive radio and dynamic spectrum access provide efficient ways to exploit the spectrum. In this sense, new users opportunistically communicate using the non-used resources of a wireless network causing little or non-existent interference.

The majority of the physical layer proposals are based on centralized multi-carrier modulations, which use spectrum sensing [1] to detect the available spectral holes over the sensed bandwidth. This information is used to tune the parameters of the multi-carrier modulation. Concerning these modulations, the proposals are focused mainly on orthogonal frequencydivision multiplexing (OFDM) [2], [3] and multi-carrier codedivision multiple access (MC-CDMA) [4]. In addition to multicarrier schemes, waveform adaptation [5] permits the new users (also known as secondary users in the literature) to dynamically adapt their transmission waveform taking into consideration the results of spectrum sensing.

Resource allocation in multi-carrier modulations requires a centralized network topology, i.e., an inefficient implementation due to backbone communications. In this sense, waveform optimization is a key enabling issue to increase the system

This work has been supported by projects TEC2013-47020-C2-2-R (COMPASS) and WINTER: TEC2016-76409-C2-1-R (AEI/FEDER, UE), and the grant FJCI-2014-2274 of the Spanish Ministry of Economy, Industry and Competitiveness. efficiency while guaranteeing the demanded bit rates. Furthermore, since the power consumption is a limitation in wireless systems, energy efficiency plays an important role in future communication networks. Concerning the latter issue, some recent works, for instance [6], [7], propose an energy-efficient power allocation with imperfect spectrum sensing.

In fact, some works, e.g. [8], [9], introduce the idea of exploiting the instantaneous interfering channels to transmit over the noise subspace, keeping the existing users (also known as primary users in the literature) free of interference. However, the use of instantaneous channel state information (CSI) is a drawback for practical implementation in real systems.

Traditional null space techniques propose transmitting over the noise subspace taking advantage of the second-order statistics of the aforementioned channel. Even though these techniques overcome the implementation issues by using the noise eigenvectors [10], [11], there is still a persisting ambiguity among the adopted noise eigenvectors due to the lack of rotational invariance, leading to non-coherent detection.

In this paper, we have considered an opportunistic communications scenario where the transmission waveform is optimized by means of the locally sampled observations correlation matrix, hence avoiding the use of an inefficient backbone. As it will be shown, the optimal waveform presents maximum spectral mismatch with the existing users and maximally white frequency response, which minimizes the possible interference to those users. Interestingly, the presented proposal exhibits rotational invariance, avoiding the noise eigenvectors ambiguity. As it will be explained, the derived solution may be seen as a dimension-spreading technique. Finally, an asymptotically efficient implementation of this strategy is presented.

\section{Problem Statement}

This section is intended to describe the waveform optimization problem in the context of opportunistic communications. Let $W$ be the sensed bandwidth. We assume that some of the radio resources are occupied by the existing users, namely the external users. In opportunistic communications, a new set of internal users of an internal wireless network want to communicate over the available radio resources, minimizing any interference on the existing external users. 
In this sense, and for the sake of simplicity, we assume that two new internal users wish to establish a point-to-point transmission based on a generic modulation signal of the form

$$
s(t)=\sum_{n} \sum_{k=1}^{K} a_{k}[n] \varphi_{k}(t-n T),
$$

being $a_{k}[n]$ any symbol from a constellation of size $P, \varphi_{k}(t)$ are the transmission waveforms to be optimized, $T$ is the symbol period, and $k$ stands for the indexing of orthogonal waveform set

$$
\mathcal{W}=\left\{\varphi_{1}(t), \ldots, \varphi_{K}(t)\right\} .
$$

The cardinality of this set is the number of available degreesof-freedom (DoF) for internal users. It is worth noting that a user can use a single DoF $K=1$, or more than one $K>1$, according to the traffic conditions. The DoF concept is adopted as it is coined by R. Gallager [12], and by D. Tse and P. Viswanath [13], i.e., the size of the set of complex numbers required to specify any particular class of signals.

That is, for band-limited, time-limited, and energy-limited signals $\varphi_{k}(t)$ in (1) the maximum DoF will be given by $N=W T$ complex dimensions. In this paper, we assume that the existing external users employ an amount of radio resources equivalent to $M<N$ in the wireless network. Hence, $K=N-M$ is the number of DoF available for opportunistic transmission between two internal users. Then, as it is previously said, the cardinality of (2) is $|\mathcal{W}|=K$.

Let $\Phi_{k}(\omega)$ denote the Fourier transform of $\varphi_{k}(t)$, and let $\phi_{s}(\omega)$ be the aggregated average power spectral density (PSD) of the external users, which is observed by any generic internal user. Ideally, the PSD of the internal users and the PSD of the external users should exhibit maximum spectral mismatch, or equivalently minimum overlap over the monitored bandwidth, which can be mathematically expressed as

$$
\varphi_{k}(t)=\underset{\varphi(t): \mathcal{F}[\varphi(t)]=\Phi(\omega)}{\arg \min } \frac{1}{2 \pi} \int_{-\pi}^{\pi} \phi_{s}(\omega)|\Phi(\omega)|^{2} \mathrm{~d} \omega
$$

where $\omega$ represents the normalized frequency, and $\mathcal{F}[x(t)]$ denotes the Fourier transform of $x(t)$. Let $\varphi_{k} \in \mathbb{C}^{N}$ be the vector representation of $\varphi_{k}(t)$, and let $\mathbf{R}_{s} \in \mathbb{C}^{N \times N}$ be the autocorrelation matrix of the external users' signal. Notice that in the time-domain, (3) becomes

$$
\boldsymbol{\varphi}_{k}=\underset{\varphi}{\arg \min } \operatorname{tr}\left(\mathbf{R}_{\mathrm{s}} \boldsymbol{\varphi} \boldsymbol{\varphi}^{\mathrm{H}}\right),
$$

being $\operatorname{tr}(\mathbf{X})$ the trace of a square matrix $\mathbf{X}$. It is worth noting that the optimization problem in (3) and (4) will be subjected to non-trivial design constraints.

Actually, the estimation of $\mathbf{R}_{s}$ performed by the internal users will suffer from estimation errors due to the effects of multipath fading, shadowing and noisy measures. Let $\mathbf{x}_{t}$ be the local observations at the internal transmitting node, and let $\mathrm{x}$ be the local observations at another arbitrary internal node. We then model the sensed autocorrelation matrix of the external users' signal at any internal node as

$$
\mathbf{R}_{s}(\mathbf{x})=\mathbf{R}_{s}\left(\mathbf{x}_{t}\right)+\boldsymbol{\Delta}\left(\mathbf{x} ; \mathbf{x}_{t}\right),
$$

where $\mathbf{R}_{s}\left(\mathbf{x}_{t}\right)$ is the autocorrelation matrix sensed at the internal transmitting node, and $\boldsymbol{\Delta}\left(\mathbf{x} ; \mathbf{x}_{t}\right)$ is a positive semidefinite matrix which reflects the DoF uncertainty with respect to the transmitter at any other location $\mathbf{x}$. We note that $\boldsymbol{\Delta}\left(\mathrm{x}_{t} ; \mathbf{x}_{t}\right)=\mathbf{0}$. The model adopted in (5) refers to a spacevariant estimation error, since the error matrix $\boldsymbol{\Delta}\left(\mathbf{x} ; \mathbf{x}_{t}\right)$ varies depending on the location of the receiving node. Henceforth, to simplify the notation, we will denote $\mathbf{R}_{s}\left(\mathbf{x}_{t}\right)=\mathbf{R}_{s}$ and $\boldsymbol{\Delta}\left(\mathbf{x} ; \mathbf{x}_{t}\right)=\boldsymbol{\Delta}$. On that account, by using (5), we may consider the worst-case cross-interference in the design problem formulated in (4), i.e.,

$$
\boldsymbol{\varphi}_{k}=\underset{\boldsymbol{\varphi}}{\arg \min } \max _{\boldsymbol{\Delta}} \boldsymbol{\varphi}^{H}\left(\mathbf{R}_{s}+\boldsymbol{\Delta}\right) \boldsymbol{\varphi} \text { s.t. }\|\boldsymbol{\Delta}\|_{F}^{2} \leq \epsilon^{2},
$$

where $\|\mathbf{X}\|_{F}^{2}=\operatorname{tr}\left(\mathbf{X} \mathbf{X}^{\mathrm{H}}\right)$ denotes the squared Frobenius norm of matrix $\mathbf{X}$, and the uncertainty on matrix $\Delta$ is upper-bounded by a certain value $\|\boldsymbol{\Delta}\|_{F}^{2} \leq \epsilon^{2}$. The Lagrangian associated to the maximization problem in (6) is given by

$$
\mathcal{L}(\boldsymbol{\Delta}, \mu)=\varphi^{H}\left(\mathbf{R}_{s}+\boldsymbol{\Delta}\right) \boldsymbol{\varphi}-\mu\left(\epsilon^{2}-\operatorname{tr}\left(\boldsymbol{\Delta} \boldsymbol{\Delta}^{\mathrm{H}}\right)\right) .
$$

Taking the gradient of (7) with respect to $\Delta$ we obtain

$$
\Delta=\frac{\epsilon}{\|\varphi\|^{2}} \varphi \varphi^{H}
$$

Regarding to (8), in an orthogonal opportunistic scenario (i.e. when $\mathbf{R}_{s}^{H} \boldsymbol{\varphi}=\mathbf{0}$ ) the interference may be reduced by means of minimizing the norm of the transmission waveform. Further on, we will refer to this approach as the minimum-norm waveform $(\mathrm{MNW})$. It is worth noting that the scenario presented in (8) is very pessimistic, since it indicates that the internal users have estimated as available the DoF occupied by the external network. Notice that it is related to a very large uncertainty and/or to monitoring techniques with a poor performance.

In essence, (8) indicates that minimizing the norm of the transmission waveform is the best that can be done to achieve the highest performance. Hence, the minimum-norm solution robustly exploits the available DoF in the worst-case scenario. This is a possible interpretation of the reason why robust spectral estimation techniques often adopt minimum-norm criteria, e.g. the seminal paper [14] and the robustness studies of the solution as particular cases of total least squares [15], in many different contexts.

At this point, we reformulate (4) taking into consideration the previous discussion about (8). Consequently, the waveform optimization problem addressed in this paper is given by

$$
\boldsymbol{\varphi}_{k}=\underset{\varphi}{\arg \min }\|\boldsymbol{\varphi}\|^{2} \text { s.t. } \mathbf{R}_{s}^{H} \boldsymbol{\varphi}=\mathbf{0} \text { and } \boldsymbol{\varphi}_{k}^{H} \mathbf{e}_{k}=1,
$$

where

$$
\mathbf{e}_{k}=[\underbrace{0 \ldots 0}_{N-k} 1 \underbrace{0 \ldots 0}_{k-1}]^{T} .
$$

The non-trivial constraint in (9) will be further optimized. It can be proved that, as stated in [15], the optimization in (9) is equivalent to a total least squares optimization. 


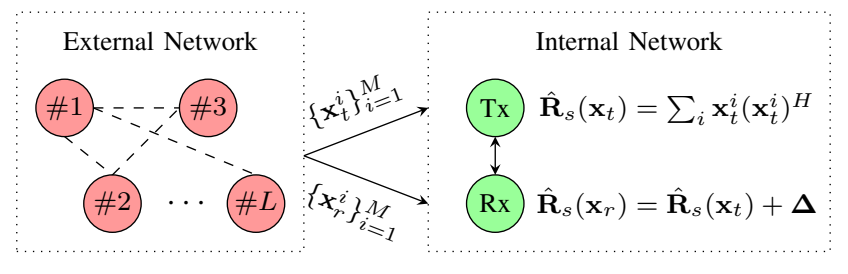

Fig. 1. Point-to-point opportunistic communication problem between two internal users exploiting the second-order statistics of the external network.

\section{Minimum-Norm WaVEForm Optimization}

The considered opportunistic scenario is depicted in Fig. 1 for a point-to-point opportunistic communications link. Based on local spectrum sensing [1] and model order selection [16], the transmitting and receiving users independently estimate the autocorrelation matrix of the external network users' aggregate signal that are denoted as follows

$$
\begin{aligned}
& \mathbf{R}_{s}\left(\mathbf{x}_{t}\right) \text { with } M_{t}=\operatorname{rank}\left(\mathbf{R}_{s}\left(\mathbf{x}_{t}\right)\right), \\
& \mathbf{R}_{s}\left(\mathbf{x}_{r}\right) \text { with } M_{r}=\operatorname{rank}\left(\mathbf{R}_{s}\left(\mathbf{x}_{r}\right)\right) .
\end{aligned}
$$

Recalling the definition of the number of available DoF, we see that $K_{t}=N-M_{t}$ and $K_{r}=N-M_{r}$ represent the available DoF estimated by the transmitter and the receiver, respectively. Ideally, $M_{t}=M_{r}=M$, with

$$
\mathbf{R}_{s}\left(\mathbf{x}_{t}\right) \approx \mathbf{R}_{s}\left(\mathbf{x}_{r}\right) \approx \mathbf{R}_{s} .
$$

However, shadowing and multipath fading may cause that the autocorrelation matrices in (11) and (12) differ in rank, leading to two possible situations

$$
\begin{aligned}
& K_{t}<K_{r}, \\
& K_{t}>K_{r} .
\end{aligned}
$$

When the transmitter underestimates the available DoF, i.e. (14), the receiver will observe a noise enhancement as it is detecting on a signal space with more DoF than the ones used by the transmitter. Otherwise, when the available DoF are overestimated, i.e. (15), the receiver will suffer from a bit-energy loss, because the transmitter uses more DoF than the ones that are expected at the receiver. In addition, in the latter case, an interference to external-network receivers may be observed. This interference is caused because certain DoF occupied by the external network can be also used as in (5). Hence, this scenario will be modeled as

$$
\mathbf{R}_{s}\left(\mathbf{x}_{r}\right)=\mathbf{R}_{s}\left(\mathbf{x}_{t}\right)+\boldsymbol{\Delta},
$$

where $\Delta$ is independent of the external network.

In the presence of white spaces, the aggregate signal of the $L$ external users is contained in a signal subspace, which is exhibited in the autocorrelation matrix of the locally noisy sampled observations. We now assume that both the transmitting and receiving internal users estimate this autocorrelation matrix $\hat{\mathbf{R}}$, which admits the following decomposition

$$
\begin{aligned}
\hat{\mathbf{R}} & =\mathbf{R}_{s}+\mathbf{R}_{n} \\
& =\mathbf{U}_{s}\left(\boldsymbol{\Lambda}_{s}+\sigma^{2} \mathbf{I}_{M}\right) \mathbf{U}_{s}^{H}+\sigma^{2} \mathbf{U}_{n} \mathbf{I}_{K} \mathbf{U}_{n}^{H}
\end{aligned}
$$

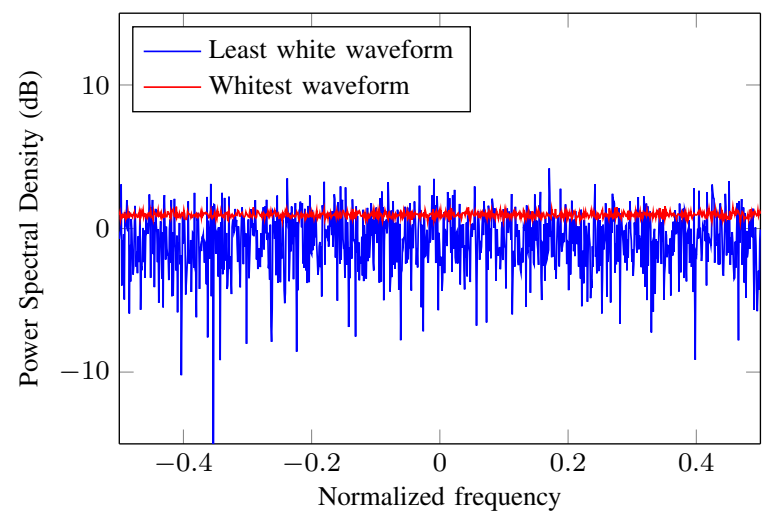

Fig. 2. PSD of two MNW according to (19). The whitest one also fulfills (20), while the least white waveform corresponds to the last iteration of the orthogonal set in (2).

where $\mathbf{R}_{s}$ and $\mathbf{R}_{n}$ are the signal and noise autocorrelation matrices, respectively. Concerning the decomposition in (17b), $\mathbf{U}_{s}$ and $\mathbf{U}_{n}$ are the eigenvectors that span the signal and noise subspaces, respectively. $\boldsymbol{\Lambda}_{s}$ is a diagonal matrix containing the $M$ signal eigenvalues, and $\sigma^{2}$ is the noise variance. Here, $\mathbf{I}_{M}$ and $\mathbf{I}_{K}$ denote the identity matrices of size $M$ and $K$, respectively.

At this point and based on (17b), it can be shown that the solution to the optimization problem (9)-(10) is equivalent to find the waveform $\varphi_{k}=\mathbf{U}_{n} \boldsymbol{\lambda}_{k}$ such that

$$
\boldsymbol{\lambda}_{k}=\underset{\boldsymbol{\lambda}_{k}}{\arg \min }\left\|\boldsymbol{\varphi}_{k}\right\|^{2} \text { s.t. } \boldsymbol{\varphi}_{k}^{H} \mathbf{e}_{k}=1,
$$

leading to

$$
\boldsymbol{\varphi}_{k}=\frac{1}{\left(\mathbf{e}_{k}^{H} \mathbf{U}_{n} \mathbf{U}_{n}^{H} \mathbf{e}_{k}\right)} \mathbf{U}_{n} \mathbf{U}_{n}^{H} \mathbf{e}_{k} .
$$

The selection of the index $k$ in (19) is highly critical, as detailed in (22), and can be further optimized following

$$
k=\underset{l \in\{1, \ldots K\}}{\arg \max } \mathbf{e}_{l}^{H} \mathbf{U}_{n} \mathbf{U}_{n}^{H} \mathbf{e}_{l} .
$$

As we can observe in (19), the optimal waveform is a certain scaled column of the noise subspace projector $\mathbf{P}=\mathbf{U}_{n} \mathbf{U}_{n}^{H}$. It is worth noting that the projector $\mathbf{P}$ is maximizing the distance between the transmitted waveforms by performing a dimension spreading. As mentioned in Sec. II, we can construct a set of $K$ orthogonal waveforms. This set can be recursively obtained by removing the already used $\mathrm{DoF}$ at each iteration from the orthogonal subspace, i.e.

$$
\begin{aligned}
\mathbf{P}_{1} & =\mathbf{U}_{n} \mathbf{U}_{n}^{H} \\
\mathbf{P}_{k}^{\perp} & =\mathbf{I}_{N}-\mathbf{P}_{k}, \\
\mathbf{P}_{k+1}^{\perp} & =\left[\begin{array}{ll}
\mathbf{P}_{k}^{\perp} & \boldsymbol{\varphi}_{k}
\end{array}\right],
\end{aligned}
$$

where $k$ indexes the iterations from 1 to $|\mathcal{W}|=K$. 


\section{A. Properties of the Minimum-Norm Solution}

Motivated by the former problem description, the waveform design addressed in this paper is given by the optimization problem (9)-(10) leading to (19)-(20). As it may be seen below, the properties exhibited by the solution (19) are analyzed.

1) Invariance to rotations: One of the main problems in orthogonal opportunistic transmission is that despite transmitter and receiver can share the same DoF, there exists ambiguity between the adopted transmission and reception vectorial basis. Yet, we show that the minimum-norm solution (19) relies on the noise subspace projector, which is invariant to right rotations, i.e. those within the noise subspace. We note that a rotational invariant detection enjoys a better detection performance than the one exhibited by non-coherent communication receivers. Let $\boldsymbol{\Xi}$ be a rotation matrix satisfying $\boldsymbol{\Xi} \boldsymbol{\Xi}^{H}=\mathbf{I}_{K}$. Thus, the right-rotated version of $\mathbf{U}_{n}, \mathbf{U}_{n} \boldsymbol{\Xi}$, satisfies $\mathbf{U}_{n} \boldsymbol{\Xi} \boldsymbol{\Xi}^{H} \mathbf{U}_{n}^{H}=\mathbf{P}$.

2) Spectral whiteness and uniform power distribution among all available DoF: It is possible to rewrite the optimization cost function in (9) as

$$
\boldsymbol{\varphi}_{k}=\underset{\varphi}{\arg \min } \frac{\|\boldsymbol{\varphi}\|^{2}}{\left|\boldsymbol{\varphi}^{H} \mathbf{e}_{k}\right|^{2}} \text { s.t. } \mathbf{U}_{s}^{H} \boldsymbol{\varphi}=\mathbf{0} .
$$

The objective function in (22) can be further expressed in the asymptotic frequency domain as

$$
\frac{\|\varphi\|^{2}}{\left|\varphi^{H} \mathbf{e}_{k}\right|^{2}}=\frac{\frac{1}{2 \pi} \int_{-\pi}^{\pi}|\Phi(\omega)|^{2} \mathrm{~d} \omega}{\left|\frac{1}{2 \pi} \int_{-\pi}^{\pi} \Phi(\omega) e^{-j 2 \pi \omega(k-1)} \mathrm{d} \omega\right|^{2}} \geq 1,
$$

where the last is due to the Cauchy-Schwarz inequality. We see that the ratio in (23) is minimized if and only if the PSD of the optimal waveforms is constant, i.e., implying that the transmitted spectrum is white. The non-trivial constraint in (9) implies the linear prediction condition stated in [14] and, thus, its polynomial roots will present an almost uniform distribution [17]. It is worth noting that the criterion in (22) and then in (23) are equivalent and both provide the whitest spectral solution. This property is also giving the fairest solution, because the internal users will inject the same amount of power in all available DoF. Hence, in case of bad conditions in monitoring the external network, the involved DoF will not receive a large amount of power, but the same as the remaining.

3) Energy efficiency: Since the minimum-norm solution presents a maximally white PSD, its shape in the time domain will be glaringly peaky. In terms of peak-to-average ratio (PAR), the proposed solution may be critical to be used in certain applications. In this sense, we propose the use of peak clipping, but subjected to orthogonality constraints in order to reduce the PAR while guaranteeing little or non-existent interference to the external network. Despite the importance of this issue, the whole study must be omitted in this presentation due to space reasons.

\section{ANALYSIS OF THE INTERFERENCE}

In the previous section, the optimal minimum-norm waveform has been derived under the assumption that the secondorder statistics are known perfectly. However, a more realistic scenario must consider the multipath fading and shadowing effects at the sensing transmitting node. As we have previously seen, the worst-case scenario is the one corresponding to a rank-one error matrix $\boldsymbol{\Delta}$ as in (8). Indeed, this is a very pessimistic scenario. A more general rank $-D$ model of the sensing error matrix is considered, i.e.,

$$
\boldsymbol{\Delta}=\sum_{d=1}^{D} \boldsymbol{\delta}_{d} \boldsymbol{\delta}_{d}^{H}
$$

where $D$ is the rank of the error matrix and $\boldsymbol{\delta}_{d} \in \mathbb{C}^{N}$, for $d=$ $1, \ldots, D$, are i.i.d. random vectors. In order to increase the reliability of the error model, the worst statistics in terms of information degradation are assumed. As it is well-known, a Gaussian interference will cause the maximum information degradation if the input noiseless signal also follows a Gaussian distribution, subject to a transmission power constraint. Therefore, we assume that

$$
\boldsymbol{\delta}_{d} \sim \mathcal{C N}\left(\mathbf{0} ; \sigma_{\delta}^{2} \mathbf{I}_{N}\right), \text { for } d=1, \ldots, D .
$$

From (25), we can easily observe that

$$
\mathbb{E}[\boldsymbol{\Delta}]=D \sigma_{\delta}^{2} \mathbf{I}_{N},
$$

and

$$
\mathbb{E}\left[\|\boldsymbol{\Delta}\|_{F}^{2}\right]=D N(N+D) \sigma_{\delta}^{4} \triangleq \epsilon^{2},
$$

where $\epsilon$ reflects the level of uncertainty. Let $\xi_{\epsilon}(D)$ denote the interference power level provided by the orthogonal opportunistic transmission to the external network as a function of the rank of error matrix $D$ and the uncertainty $\epsilon$. Then, it follows from (26)-(27) that

$$
\begin{aligned}
\xi_{\epsilon}(D) & =\mathbb{E}\left[\boldsymbol{\varphi}^{H} \boldsymbol{\Delta} \boldsymbol{\varphi}\right] \\
& =\sigma_{\delta}^{2} D\|\boldsymbol{\|}\|^{2} \\
& =\epsilon \sqrt{\frac{D}{N(N+D)}} .
\end{aligned}
$$

The undesired induced interference power depends on the rank of the error matrix $D$, the total number of $\operatorname{DoF} N$, and it is proportional to the uncertainty level $\epsilon$, as it is depicted in Fig. 3.

\section{ASYMPTOTIC BEHAVIOR}

Throughout the paper and for the sake of simplicity, the problem analysis and the discussion of the solution are done in terms of eigenvectors. According to Szegö's limit theorems, a Toeplitz matrix as (17a) is asymptotically equivalent to a circulant matrix [18]. Hence, the eigenfactorization of (17a) asymptotically leads to a Fourier matrix-based decomposition

$$
\hat{\mathbf{R}} \underset{N \rightarrow \infty}{\longrightarrow} \mathbf{F} \boldsymbol{\Lambda} \mathbf{F}^{H},
$$

being $\mathbf{F}$ the normalized Fourier matrix, and $\boldsymbol{\Lambda}$ the nondecreasing eigenvalues matrix. According to (29), we can find the set of available DoF, which corresponds to the set of frequency bins with null spectral contribution. Hence,

$$
\mathbf{P}=\sum_{n \in \mathcal{N}} \mathbf{f}_{n} \mathbf{f}_{n}^{H}
$$




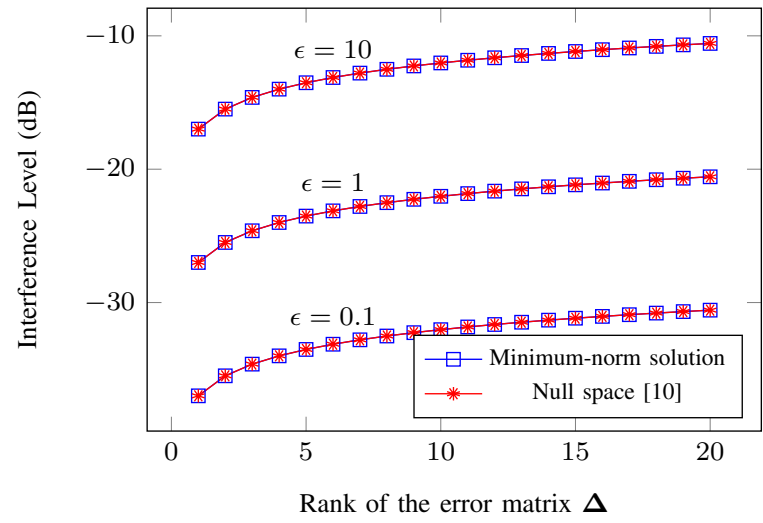

Fig. 3. Interference level provided by the optimal solution in (19) for different uncertainty levels in benchmarking to null space method [10].

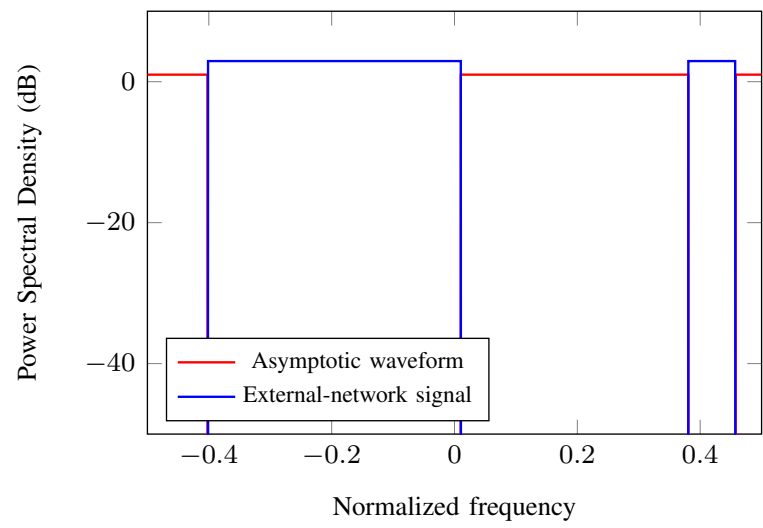

Fig. 4. Power Spectral Density of the asymptotic waveform and the externalnetwork spectral occupation with a generic scaling.

denoting $\mathcal{N}$ the set of the available frequency bins, i.e. the noise subspace, and

$$
\mathbf{f}_{n}=\frac{1}{\sqrt{N}}\left[1 \omega_{n} \cdots \omega_{n}^{N-1}\right]^{T},
$$

being $\omega_{n}$ the primitive $n$-th root of the unity. Following the methodology described in (19)-(20), the minimum-norm solution asymptotically behaves as a linear combination of the non-occupied frequency bins (see Fig. 4). This implies a direct link of the results presented in this paper with highefficient DFT-based multi-carrier solutions. Furthermore, when the impact of the channel is considered, induced circulant channel matrices can be obtained by the use of a conventional cyclic prefix, i.e.,

$$
\varphi(n) \circledast h(n) \underset{\mathcal{F}}{\rightarrow} \mathbf{F}^{H}(\mathbf{h} \odot \mathbf{F} \boldsymbol{\varphi})=\mathbf{F}^{H} \mathbf{H F} \boldsymbol{\varphi},
$$

where $\mathbf{H}$ is a diagonal matrix whose diagonal $\mathbf{h}$ contains the spectral contribution of the channel, and $\circledast$ and $\odot$ denote the circular convolution and the Schur-Hadamard product, respectively. This property is of paramount importance, since known techniques in multiplicative channel transmission can be used to mitigate the effects of the interference and exploit any potential diversity gain provided by the channel memory. It is worth noting that, in the asymptotic case, the use of the eigendecomposition is avoided, which implies an improvement in terms of computational efficiency.

\section{CONCLUSIONS}

This paper has addressed the minimum-norm waveform optimization problem based on the second-order statistics of the external network. The presented technique is a dimensionspreading solution, and therefore presents a processing gain and robustness against interference. The derived waveforms exhibit the properties of white spectrum, rotationally invariance, and robustness to the worst-case subspace mismatch, which are both numerically and theoretically discussed together with the interference. Finally, its asymptotic performance is reported.

\section{REFERENCES}

[1] S. Haykin, D. J. Thomson, and J. H. Reed, "Spectrum sensing for cognitive radio," Proc. IEEE, vol. 97, no. 5, pp. 849-877, May 2009.

[2] B. Farhang-Boroujeny and R. Kempter, "Multicarrier communication techniques for spectrum sensing and communication in cognitive radios," IEEE Commun. Mag., vol. 46, no. 4, pp. 80-85, 2008.

[3] T. Weiss and F. Jondral, "Spectrum pooling: an innovative strategy for the enhancement of spectrum efficiency," IEEE Commun. Mag., vol. 42, no. 3, pp. S8-14, 2004

[4] A. Attar, M. R. Nakhai, and A. H. Aghvami, "Cognitive radio transmission based on direct sequence MC-CDMA," IEEE Trans. Wireless Commun., vol. 7, no. 4, pp. 1157-1162, April 2008.

[5] Z. Tian, G. Leus, and V. Lottici, "Joint dynamic resource allocation and waveform adaptation for cognitive radio," IEEE J. Sel. Areas Commun., vol. 29, no. 2, pp. 443-454, 2011.

[6] G. Ozcan, M. C. Gursoy, N. Tran, and J. Tang, "Energy-efficicient power allocation in cognitive radio systems with imperfect spectrum sensing," IEEE J. Sel. Areas Commun., vol. 34, no. 2, pp. 3466-3481, December 2016.

[7] A. Alabbasi, Z. Rezki, and B. Shihada, "Energy efficient resource allocation for cognitive radios: A generalized sensing analysis," IEEE Trans. Wireless Commun., vol. 14, no. 5, pp. 2455-2469, May 2015.

[8] L. Zhang, Y. C. Liang, Y. Xin, and H. V. Poor, "Robust cognitive beamforming with partial channel state information," IEEE Trans. Wireless Commun., vol. 8, no. 8, pp. 4143-4153, August 2009.

[9] Y. Zhang, E. DallAnese, and G. B. Giannakis, "Distributed optimal beamformers for cognitive radios robust to channel uncertainties," IEEE Trans. Signal Process., vol. 60, no. 12, pp. 6495-6508, Dec 2012.

[10] H. Yi, H. Hu, Y. Rui, K. Guo, and J. Zhang, "Null space-based precoding scheme for secondary transmission in a cognitive radio MIMO system using second-order statistics," Proc. IEEE Int. Conf. on Commun. (ICC), June 2009.

[11] S. Sodagari, "On effects of imperfect channel state information on null space based cognitive MIMO communication," in 2015 Int. Conf. on Comp., Netw. and Commun. (ICNC), Feb 2015, pp. 438-444.

[12] R. G. Gallager, Information Theory and Reliable Communications. John Wiley \& Sons, 1968.

[13] D. Tse and P. Viswanath, Fundamentals of Wireless Communications. Cambridge University Press, 2005.

[14] R. Kumaresan and D. W. Tufts, "Estimating the parameters of exponentially damped sinusoids and pole-zero modeling and noise," IEEE Trans. Acoust., Speech and Signal Process., vol. ASSP-30, no. 6, pp. 833-840, December 1982.

[15] E. M. Dowling and R. D. DeGroat, "The equivalence of the total least squares and minimum norm methods," IEEE Trans. Signal Process., vol. 39, no. 8, pp. 1891-1892, August 1991.

[16] P. Stoica and Y. Selen, "Model-order selection: a review of information criterion rules," IEEE Signal Process. Mag., vol. 21, no. 4, pp. 36-47, July 2014.

[17] R. Kumaresan, "On the zeros of the linear prediction-error filter for deterministic signals," IEEE Trans. Acoust., Speech and Signal Process., vol. 31, no. 1, pp. 217-220, February 1983.

[18] R. M. Gray, Toeplitz and Circulant Matrices: A Review. New York, NY: Hanover/Now, 2006. 